\title{
Transient optical polarization response of aluminium at an interband transition
}

\author{
R Wilks and R J Hicken \\ School of Physics, University of Exeter, Stocker Road, Exeter, Devon EX4 4QL, UK \\ E-mail: R.J.Hicken@exeter.ac.uk
}

Received 26 January 2004

Published 11 June 2004

Online at stacks.iop.org/JPhysCM/16/4607

doi:10.1088/0953-8984/16/25/018

\begin{abstract}
Femtosecond optical pump-probe measurements have been performed upon a $500 \AA$ polycrystalline Al film at an interband transition at a wavelength of $800 \mathrm{~nm}$. The dependence of the intensity and polarization of the reflected probe pulse upon the polarization of the pump pulse has been determined. A sharp peak was observed in the polarization signal at zero time delay that contains contributions from both the specular inverse Faraday effect (SIFE) and the specular optical Kerr effect (SOKE). The SIFE and SOKE are associated with the optical orientation of angular and linear momentum, respectively. The dependence of the peak height upon the pump polarization has allowed us to deduce values of $\chi_{x x y y}=(1+0.6 \mathrm{i}) \times 10^{-8} \mathrm{rad} \mathrm{cm}^{3} \mathrm{erg}^{-1}$ and $\chi_{x y y x}=(0.5-0.1 \mathrm{i}) \times 10^{-8} \mathrm{rad} \mathrm{cm}^{3} \mathrm{erg}^{-1}$ for the non-vanishing components of the local cubic susceptibility tensor. A distortion of the peak was observed when the pump was elliptically polarized. Its detailed shape shows that the characteristic relaxation time for the SOKE is about 30 fs longer than that for the SIFE in measurements of ellipticity. This suggests a similar difference in the relaxation times for the angular and linear momentum of the optically excited hot electrons. The initial peak was generally followed by a tail that decayed with a time constant of several picoseconds, which we attribute to an ultrafast anisotropic excitation of the lattice.
\end{abstract}

\section{Introduction}

Femtosecond optical pump-probe experiments provide unique access to the electronic relaxation times that underlie transport processes in condensed matter systems. Measurements of transient reflectivity reveal the times taken for hot electrons to thermalize and for the thermalized electrons to reach thermal equilibrium with the lattice [1]. Measurements of transient polarization allow the excitation and relaxation of transient magnetization $[2,3]$ and 
optical anisotropy [4] to be explored. The polarization of the probe pulse is described by the non-linear susceptibility tensor, with the lowest order (cubic) processes involving two pump photons and one probe photon. For an isotropic non-gyrotropic material, such as a polycrystalline metal, there are contributions from the specular optical Kerr effect (SOKE) and the specular inverse Faraday effect (SIFE) [5]. The SOKE (SIFE) may be excited by a linearly (circularly) polarized pump beam, which transfers linear (angular) momentum to electrons in the surface of the sample, and leads to a transient linear (circular) birefringence and dichroism. The transient polarization signal thus contains information about the rate of relaxation of the linear and angular momentum of the excited electrons. In the case of metals the relevant relaxation times are of the order of $10 \mathrm{fs}$. Therefore in measurements made with $100 \mathrm{fs}$ pulses, the SIFE and SOKE are expected to appear as a peak in the transient polarization signal with width determined by that of the laser pulse. However, if the electron momentum is coupled to another sub-system within the sample that relaxes more slowly, the peak may be followed by a tail from which the characteristic relaxation times of that sub-system may be determined. For example, a linear displacement of the electrons in the surface of a metal may cause the lattice to become strained. The resulting lattice dynamics can then be detected through the associated transient birefringence (SOKE).

Aluminium exhibits an interband transition between parallel bands at the Brillouin zone boundary [6] that leads to a reduced reflectivity and a piezo-optical resonance at a wavelength of $800 \mathrm{~nm}$ [7]. Pump-probe measurements of transient reflectivity revealed an electron-phonon relaxation time of $0.6 \mathrm{ps}$ [8] and acoustic echoes associated with the multiple reflection of a thermoelastic pulse within thin film samples $[9,10]$. Using larger pump fluences, Guo et al [11] demonstrated an ultrafast melting of the Al surface. The interband optical transition was interpreted as a bonding to anti-bonding transition that deforms the lattice and induces a collapse of the band structure. We recently presented transient optical rotation measurements at $800 \mathrm{~nm}$ that revealed a peak and a tail with a relaxation time of about $6 \mathrm{ps}$ [12]. The dependence of the amplitude of these features upon the pump polarization was found to be characteristic of the SOKE. The tail feature was hence attributed to a pump-induced lattice excitation rather than to optical orientation of spin [13], which should instead exhibit a SIFE-like dependence upon the pump polarization. Although the rotation signal showed no SIFE contribution, the possibility of a SIFE signal in the transient ellipticity response was not explored.

In this paper we present a full determination of the transient polarization response of polycrystalline $\mathrm{Al}$ at the interband transition. We investigate how both the rotation and ellipticity of the probe pulse change as the pump pulse assumes linear, elliptical and circular polarization states. We obtain values for all components of the optical susceptibility tensor that give rise to the SIFE and SOKE peaks and quantify a small time delay that occurs between these peaks. We confirm that the longer-lived transient in the polarization signals occurs due to SOKE alone and find that as many as four different relaxation times are required to fully describe the transient polarization response.

\section{Experimental details}

Measurements were performed upon an $\mathrm{Al} / \mathrm{Si}$ (100) sample fabricated by magnetron sputtering. $\mathrm{X}$-ray diffraction confirmed that the $\mathrm{Al}$ had a polycrystalline fcc structure with no preferred orientation normal to the film plane. Grazing incidence measurements revealed a film thickness of $450 \AA$ as compared to a value of $500 \AA$ obtained from the in situ quartz crystal thickness monitor. The film has a thickness equal to about three optical skin depths and so is effectively semi-infinite in terms of the penetration of the optical field. The finite film thickness may affect the propagation of hot electrons away from the surface region [14], yet such considerations are 
beyond the scope of the present study. Details of the pump-probe measurements have been given previously [12]. In the present study the incident probe beam was p-polarized and the intensity and rotation of the reflected beam were recorded simultaneously with an optical bridge detector. Alternatively, by inserting a quarter waveplate in front of the detector, ellipticity could be measured instead of rotation. The angles of incidence of the pump and probe beams were $25^{\circ}$ and $47^{\circ}$ respectively. Sets of measurements were acquired with a pump fluence of about $1 \mathrm{~mJ} \mathrm{~cm}^{-2}$ as the pump polarization was varied by one of two methods. In the first method, the pump beam was made circularly polarized and then passed through a polarizer so that the plane of polarization could be varied continuously while leaving the intensity unchanged. In the second set the pump was made p-polarized and then passed through a quarter waveplate. As the waveplate was rotated the pump polarization varied continuously from linear $(\mathrm{p})$ to circular via an intermediate elliptical polarization state.

For a transient process characterized by a single relaxation time $\tau$, the form of the polarization signal $S(t)$ observed in the pump-probe experiment may be obtained by convolving the function $\exp (-t / \tau)$, in which $t$ is the time delay, with the pump and probe pulse profiles. In order to fit the data that will be presented later we assume that as many as three separate transient processes contribute to the measured polarization signal and that the first has a relaxation time much smaller than the pulse width. Assuming that the pulse intensity has a Gaussian profile with full width half maximum of $2 \sqrt{2 \ln 2} w$, then $S(t)$ has the form

$$
\begin{aligned}
S(t)=A \exp ( & \left.-\frac{t^{2}}{4 w^{2}}\right)+\frac{B}{2} \exp \left(\frac{w^{2}}{\tau_{1}^{2}}-\frac{t}{\tau_{1}}\right)\left(1-\operatorname{erf}\left(\frac{w}{\tau_{1}}-\frac{t}{2 w}\right)\right) \\
+ & \frac{C}{2} \exp \left(\frac{w^{2}}{\tau_{2}^{2}}-\frac{t}{\tau_{2}}\right)\left(1-\operatorname{erf}\left(\frac{w}{\tau_{2}}-\frac{t}{2 w}\right)\right)+D,
\end{aligned}
$$

where erf is the Gaussian error function, $A, B$ and $C$ are the amplitudes of the three transient processes, $\tau_{1}$ and $\tau_{2}$ are the characteristic relaxation times of the second and third processes, and $D$ is a constant offset.

The amplitudes of the transient rotation and ellipticity may be related to the cubic components of the nonlinear susceptibility tensor. Expressions have been presented for the case of continuous wave pump and probe beams at normal incidence to the sample surface [5]. They may be used to describe the amplitude of the Gaussian peak in equation (1) since both the pump and probe beams are strongly refracted towards the surface normal in our experiments, and because we expect the width of the pump and probe pulses to be large relative to the characteristic relaxation time associated with the peak. For an isotropic non-gyrotropic medium in which non-local effects can be neglected, the incoherent part of the polarization response is determined by the components of the susceptibility tensor $\chi_{x x y y}$ and $\chi_{x y y x}$. Writing the rotation and ellipticity of the incident pump and probe beams as $\left(\theta_{1}, \eta_{1}\right)$ and $\left(\theta_{2}, \eta_{2}\right)$ respectively, and since the incident probe beam is linearly polarized $\left(\eta_{2}=0\right)$, the change in polarization of the probe beam is given by

$$
\begin{gathered}
\left(\begin{array}{c}
\Delta \theta_{2} \\
\Delta \eta_{2}
\end{array}\right)=-\frac{32 \pi^{2} I_{\text {pump }}}{c|1+n|^{2}}\left\{\sin 2\left(\theta_{1}-\theta_{2}\right) \cos 2 \eta_{1}\left(\begin{array}{l}
\operatorname{Re} \\
\operatorname{Im}
\end{array}\right) \frac{\chi_{x x y y}+\chi_{x y y x}}{n\left(1-n^{2}\right)}\right. \\
\left.+\sin 2 \eta_{1}\left(\begin{array}{c}
-\operatorname{Im} \\
\operatorname{Re}
\end{array}\right) \frac{\chi_{x x y y}-\chi_{x y y x}}{n\left(1-n^{2}\right)}\right\},
\end{gathered}
$$

in which $I_{\text {pump }}$ is the intensity of the pump beam, $c$ is the speed of light in vacuum and $n$ is the complex refractive index of the medium. The first term in the sum describes the contribution due to the SOKE while the second describes that due to the SIFE. In the first configuration, 
setting $\theta_{2}=0$ for the p-polarized probe, and $\eta_{1}=0$ since the pump is linearly polarized, equation (2) reduces to

$$
\left(\begin{array}{c}
\Delta \theta_{2} \\
\Delta \eta_{2}
\end{array}\right)=\sin 2 \theta_{1}\left(\begin{array}{l}
\operatorname{Re} \\
\operatorname{Im}
\end{array}\right) F_{+} .
$$

In the second configuration, if $\phi$ denotes the angle between the fast axis of the quarter waveplate and the plane of incidence, it is easily shown that

$$
\left(\begin{array}{c}
\Delta \theta_{2} \\
\Delta \eta_{2}
\end{array}\right)=\frac{1}{2} \sin 4 \phi\left(\begin{array}{l}
\operatorname{Re} \\
\mathrm{Im}
\end{array}\right) F_{+}+\sin 2 \phi\left(\begin{array}{c}
-\mathrm{Im} \\
\mathrm{Re}
\end{array}\right) F_{-},
$$

where

$$
F_{ \pm}=-\frac{32 \pi^{2} I_{\mathrm{pump}}}{c|1+n|^{2}}\left\{\frac{\chi_{x x y y} \pm \chi_{x y y x}}{n\left(1-n^{2}\right)}\right\} .
$$

The quantities $F_{+}$and $F_{-}$represent the amplitudes of the SOKE and SIFE respectively. The first configuration may be used to isolate the SOKE while the second configuration contains contributions due to both the SIFE and SOKE. The SIFE may be isolated in the second configuration when $\phi=(2 m+1) \pi / 4$ and $m$ is an integer, that is, when the pump is circularly polarized.

\section{Results}

The dependence of the transient reflectivity, rotation and ellipticity signals upon pump polarization is shown in figures 1 and 2 . Additional measurements of reflectivity and rotation were made as the average unchopped pump power was varied from 10 to $200 \mathrm{~mW}$. The shape of the curves was unchanged while the amplitude of the signals varied linearly with the pump power, confirming that the signals are associated with a cubic optical non-linearity. The transient reflectivity curves in figures 1(a) and 2(a) show an initial peak superimposed upon a rapidly rising background. The full width at half maximum (FWHM) of the peak is close to $170 \mathrm{fs}$ as expected from equation (1) for instantaneous relaxation and a pulse width of $120 \mathrm{fs}$, and the peak centre has been used to define zero time delay between the pump and the probe. After the first picosecond the transient reflectivity was found to reach a maximum after 6-7 ps, as previously reported [10]. Reflection of the thermo-acoustic transient within the thickness of the film [9] leads to weak oscillations, with period of approximately $13 \mathrm{ps,} \mathrm{superimposed}$ upon a slower relaxation with a time constant of 132 ps. However, only the height of the initial peak was found to be sensitive to the pump polarization.

In the first measurement configuration, the reflectivity signal at long time delays was found to vary by up to $22 \%$ as the polarizer was rotated through $\pm 90^{\circ}$. A variation of $56 \%$ was observed in the second configuration as the quarter waveplate was rotated through $\pm 90^{\circ}$. Using the Fresnel formulae for $r_{\mathrm{ss}}$ and $r_{\mathrm{pp}}$, the amount of energy absorbed from the pump is expected to change by about $3 \%$ as the pump polarization is changed from $\mathrm{p}$ to $\mathrm{s}$. The observed amplitude variation most probably occurs due to a lack of parallelism between the surfaces of the optical component that is being rotated, which causes the focused pump spot to move slightly relative to that of the probe. In each measurement configuration, the reflectivity, rotation and ellipticity scans have therefore been normalized by the same factor so that the reflectivity signal at large positive delay is the same for all settings of the optical component that is being rotated.

For the first configuration, in which the pump beam is always linearly polarized, figure 1 shows that the peak in the reflectivity signal has maximum amplitude when the pump is $\mathrm{p}$ polarized $\left(\theta_{1}=0^{\circ}\right)$ and vanishes when the pump is s-polarized $\left(\theta_{1}= \pm 90^{\circ}\right)$. Additional 


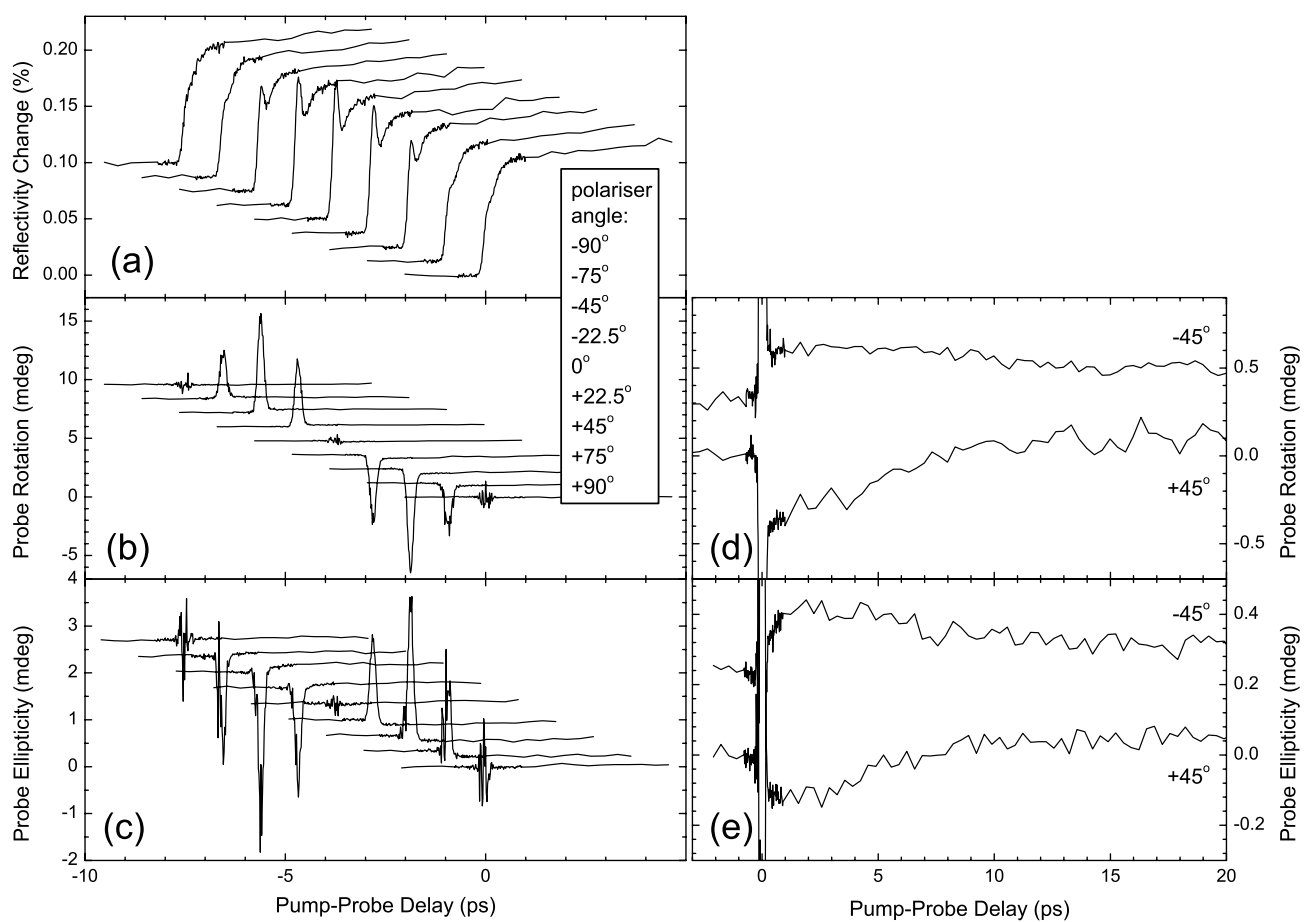

Figure 1. Measurements of (a) reflectivity, (b) rotation, and (c) ellipticity are shown for the first measurement configuration. The pump polarization was varied from $\mathrm{s}\left(\theta_{1}=-90^{\circ}\right)$ through $\mathrm{p}$ $\left(\theta_{1}=0^{\circ}\right)$ to $\mathrm{s}\left(\theta_{1}=+90^{\circ}\right)$. The curves are horizontally and vertically offset for clarity. Panels (d) and (e) show magnified views of the rotation and ellipticity curves from panels (b) and (c) respectively, for which the plane of polarization of the pump and probe lie $45^{\circ}$ apart $\left(\theta_{1}= \pm 45^{\circ}\right)$. The curves are vertically offset for clarity.

measurements made with an s-polarized probe beam confirmed that the height of the peak depends only upon the relative orientation of the pump and probe electric field vectors. In the second configuration, where the pump has elliptical polarization, figure 2 shows that the peak height is largest for the p-polarized pump $\left(\theta_{1}=0^{\circ}, \pm 90^{\circ}\right)$ and smallest, but non-vanishing, for circular pump polarization $\left(\theta_{1}= \pm 45^{\circ}\right)$.

In general the transient rotation and ellipticity signals were found to consist of a sharp peak at zero time delay, again with an FWHM close to $170 \mathrm{fs}$, and a small tail that became immeasurably small for time delays greater than 20 ps. In figure 1, where the pump is linearly polarized, both rotation and ellipticity exhibit a sharp peak at zero time delay followed by a distinct tail of smaller amplitude. The amplitude of the peak and tail exhibit a similar dependence upon the pump polarization. The signal vanishes when the pump is s- or ppolarized $\left(\theta_{1}=0^{\circ}\right.$ and $\pm 90^{\circ}$ respectively), and is greatest when the plane of polarization of the pump and probe lie $45^{\circ}$ apart. The rapid oscillations that are sometimes observed around zero time delay are largely the result of interference of the probe with light diffusely scattered from the pump beam.

In figure 2, where the pump is elliptically polarized, the rotation and ellipticity signals exhibit qualitatively different dependences upon the setting of the quarter waveplate. The rotation signal again contains a peak at zero time delay followed by a smaller tail. The peak vanishes when the pump has linear polarization $\left(\phi=-90^{\circ}, 0^{\circ}, 90^{\circ}\right)$ and its height appears to vary with $\phi$ with a period of $90^{\circ}$. The tail exhibits a similar dependence upon $\phi$ but is 


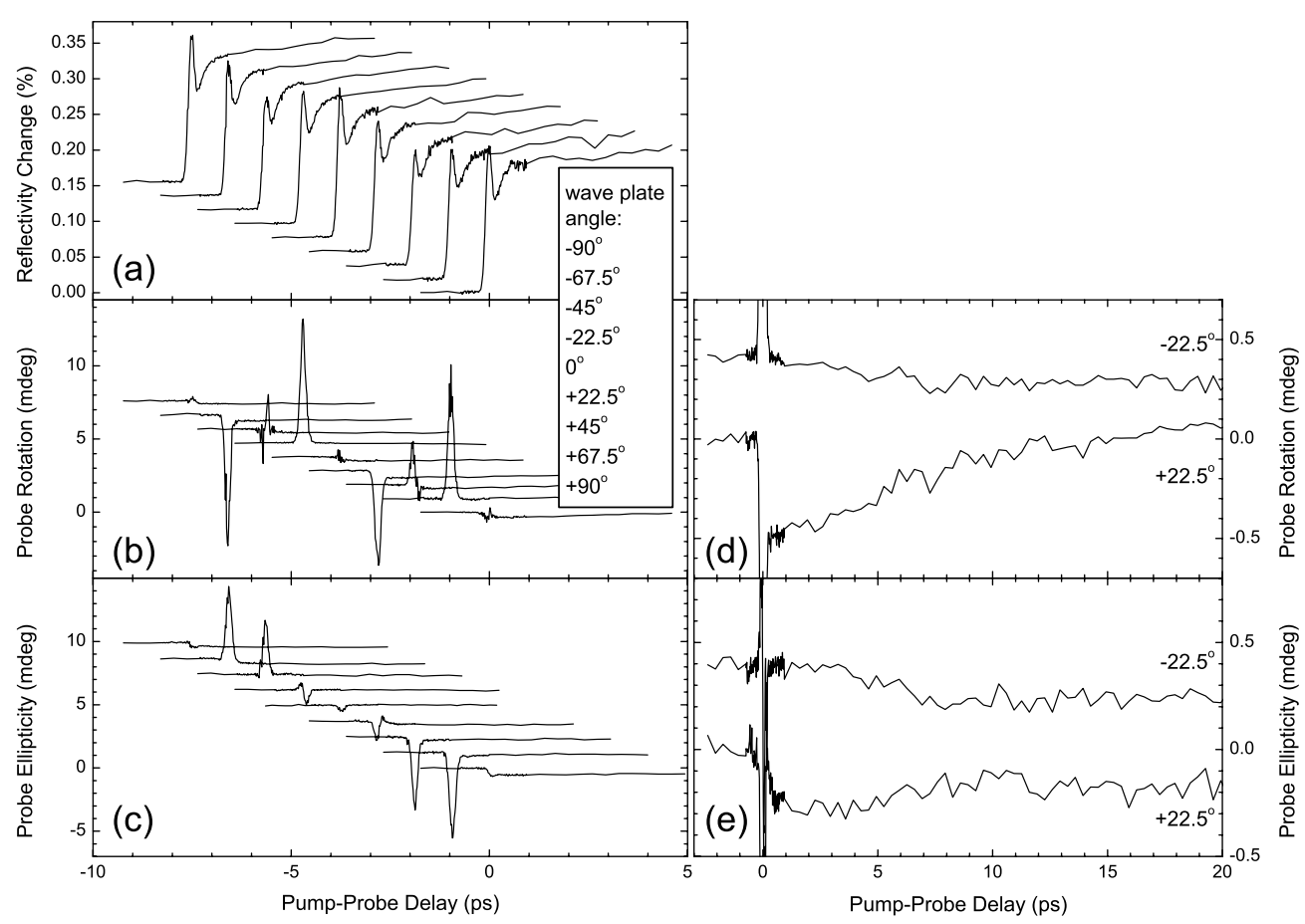

Figure 2. Measurements of (a) reflectivity, (b) rotation, and (c) ellipticity are shown for the second measurement configuration. The pump polarization was varied from $\mathrm{p}\left(\phi=-90^{\circ}\right)$ through left circular $\left(\phi=-45^{\circ}\right)$, to $\mathrm{p}\left(\phi=0^{\circ}\right)$, to right circular $\left(\phi=+45^{\circ}\right)$, to $\mathrm{p}\left(\phi=+90^{\circ}\right)$. The curves are horizontally and vertically offset for clarity. Panels (d) and (e) show magnified views of selected rotation and ellipticity curves from panels (b) and (c) respectively for the case of an elliptically polarized pump $\left(\phi= \pm 22.5^{\circ}\right)$. The curves are vertically offset for clarity.

partly obscured by a small positive step at zero time delay, due to reflectivity breakthrough that results from a small misalignment of the probe beam polarization [12]. In figure 2(b) this is most clearly seen when the pump has linear polarization $\left(\phi=0^{\circ}, \pm 90^{\circ}\right)$, and the peak and tail disappear. In figures 2(d) and (e) the tail is clearly seen for $\phi=+22.5^{\circ}$ but is obscured by the reflectivity breakthrough for $\phi=-22.5^{\circ}$. The peak in the ellipticity signal is smallest for linear pump polarization. We suspect that it does not completely vanish either because of a small misalignment of the plane of polarization of the pump beam, or because of birefringence in the focusing optics that causes the pump to have a small ellipticity. However, the peak remains large when the pump is circularly polarized, and has maximum height when $\phi= \pm 67.5^{\circ}$. When $\phi= \pm 22.5^{\circ}$ the peak is smaller and has a qualitatively different bipolar shape, with both positive and negative deviations from the signal level at negative time delay. The ellipticity data also contain a step-shaped background due to reflectivity breakthrough that again obscures the tail when $\phi=-22.5^{\circ}$.

\section{Analysis}

In order to quantify the variation of the heights of the peaks and tails in the rotation and ellipticity data, each scan has been fitted with a reduced form of equation (1) for time delays between -0.6 and $+0.8 \mathrm{ps}$. The amplitude of the second tail term, $C$, was set to zero and the value of $\tau_{1}$ was set to infinity. Clearly the bipolar curves at $\phi= \pm 22.5^{\circ}$ in figure 2(c) will 


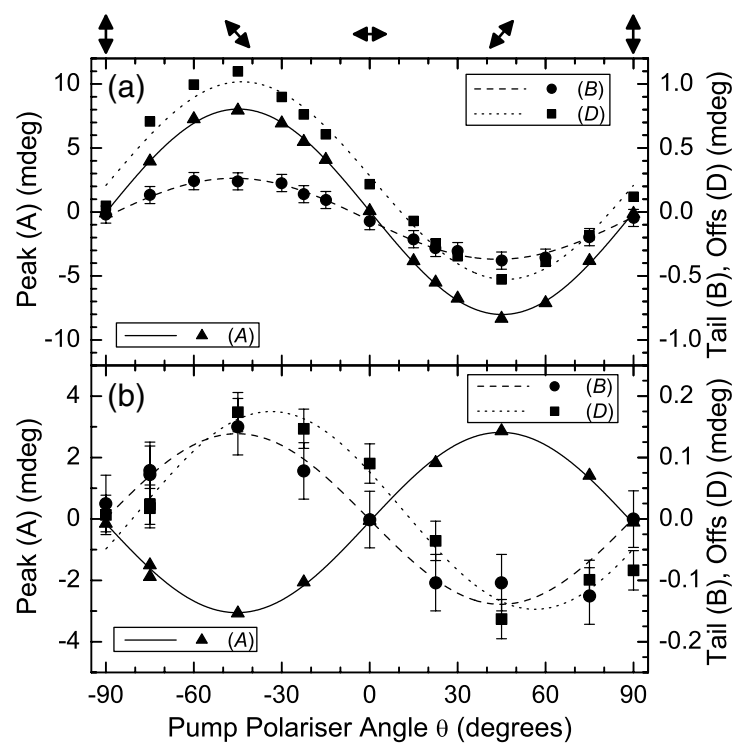

Figure 3. The symbols show fitted values of the parameters $A, B$ and $D$ as defined in equation (1). The values in the upper (a) and lower panels (b) were fitted to the transient rotation and ellipticity signals respectively obtained in the first measurement configuration. Error bars are plotted where the errors from the fit were larger than the symbol size. The curves show the fit of equation (3) to the symbols. The pump polarization is shown schematically above the figure.

not be well fitted, but they are included for consistency. The fitted values have been plotted in figures 3 and 4 .

The variation of the peak height in figures 3 and 4 has been fitted by equations (3) and (4). In figure 3 a single sinusoid with period of $180^{\circ}$ was fitted to the peak, tail and offset data. In figure 4 two sinusoids with periods of $90^{\circ}$ and $180^{\circ}$ were used. A phase offset and a vertical offset were used in fitting each sinusoid. However, very similar fits were obtained for the peak data when the offsets were instead set to zero. There is a clear vertical offset of the fitted tail amplitude (B) in figure 4 that is the result of reflectivity breakthrough. The angular dependence of the peak and tail are seen to be similar except in figure 4(b), where the variation of the tail height is smallest and the data are not well fitted by the two sinusoids. The offset parameter $(D)$ generally has a different angular dependence to the peak and tail. This is unsurprising if the offset is due to diffusely scattered pump light and is unrelated to the non-linear optical response of the sample. The fit to figure 3 yields $F_{+}=-8.0+2.9 \mathrm{i}$ mdeg while that to figure 4 yields $F_{+}=-14.2+5.6 \mathrm{i}$ mdeg and $F_{-}=-4.5-0.57 \mathrm{i}$ mdeg. Since the value of $I_{\text {pump }}$ in the first measurement configuration was half that in the second configuration, the two values of $F_{+}$are seen to be in good quantitative agreement. The values of $F_{ \pm}$obtained in the second measurement configuration have been used to estimate the values of $\chi_{x x y y}$ and $\chi_{x y y x}$. Taking $I_{\text {pump }}=9 \times 10^{16} \mathrm{erg} \mathrm{s}^{-1} \mathrm{~cm}^{-2}$ we obtain $\chi_{x x y y}=(1+0.6 \mathrm{i}) \times 10^{-8} \mathrm{rad} \mathrm{cm}^{3} \mathrm{erg}^{-1}$ and $\chi_{x y y x}=(0.5-0.1 \mathrm{i}) \times 10^{-8} \mathrm{rad} \mathrm{cm}^{3} \mathrm{erg}^{-1}$.

Since $\operatorname{Im} F_{+}$and $\operatorname{Re} F_{-}$have opposite signs, equation (4), predicts that the SIFE and SOKE make contributions of opposite sign to the initial ellipticity peak when $\phi= \pm 22.5^{\circ}$. This suggests that the bipolar peak shapes observed at $\phi= \pm 22.5^{\circ}$ in figure 2 occur because the SIFE and SOKE have a slightly different time dependence. Before this conjecture can be explored it is first necessary to remove the small residual peak and the offset due to reflectivity breakthrough that are most clearly seen at $\phi=0^{\circ}$ and $\pm 90^{\circ}$ in figure 2. Equation (4) shows 


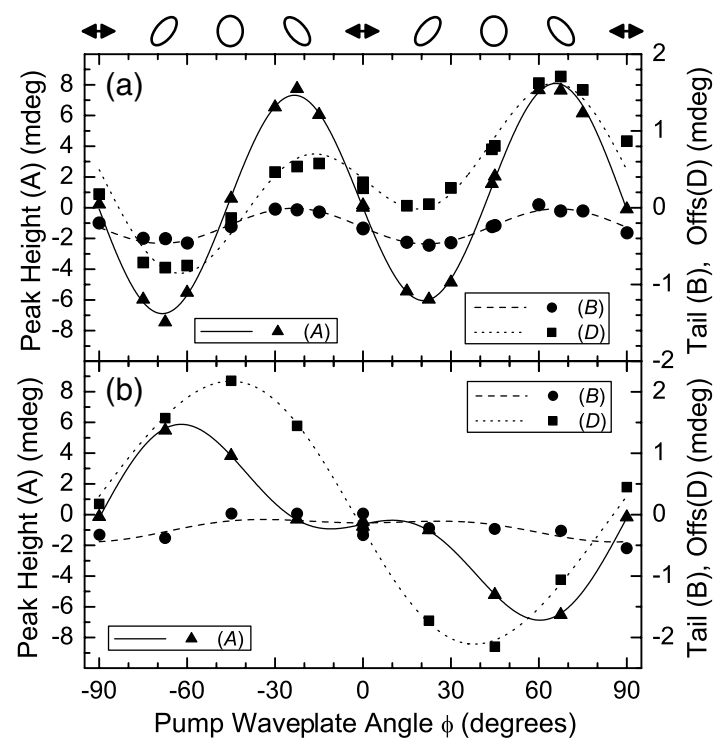

Figure 4. The symbols show fitted values of the parameters $A, B$ and $D$ as defined in equation (1). The values in the upper (a) and lower panels (b) were fitted to the transient rotation and ellipticity signals respectively obtained in the second measurement configuration. The errors from the fit are smaller than the symbol size. The lines show the fit of equation (4) to the symbols. The pump polarization is shown schematically above the figure.

that both the SIFE and SOKE contributions change sign when the sign of $\phi$ is reversed. Therefore the difference of a pair of traces at $+\phi$ and $-\phi$, should isolate the SIFE and SOKE while suppressing background contributions. The difference divided by two of pairs of scans acquired at $\pm 22.5^{\circ}, \pm 45^{\circ}$ and $\pm 67.5^{\circ}$ have been plotted in figure 5. From equation (4), the difference of signals at $\pm 45^{\circ}$ (trace (b)) is expected to result from the SIFE alone. The difference of signals at $\pm 22.5^{\circ}$ and $\pm 67.5^{\circ}$ (traces (a) and (c) respectively) should contain contributions from both the SIFE and the SOKE. Furthermore, the sum and difference of traces (a) and (c) should isolate the SIFE and the SOKE respectively. The sum of traces (a) and (c) multiplied by $\sqrt{2}$, curve (d), appears to be identical to trace (b), as one would expect. The difference of traces (c) and (a) is shown as trace (e). Comparing traces (d) and (e), the centre of the SOKE curve (e) is found to be delayed by 27 fs relative to the centre of the SIFE curve (d). This delay fully accounts for the bipolar shape of the traces observed at $\phi= \pm 22.5^{\circ}$.

From figures 1 and 2, the dependence of the tail amplitude upon the pump polarization is seen to have a clear symmetry except in the case of ellipticity in the second measurement configuration. This symmetry allows the reflectivity breakthrough to be subtracted, in the same manner as described for the peaks in the previous paragraph, so that the tail may be more clearly seen. The difference of signals at $\phi= \pm 45^{\circ}$ in figure 1 should isolate a tail that varies with pump polarization in the same manner as the SOKE. Traces (a) and (c) in figure 6 show the difference signals obtained from the rotation and ellipticity data respectively. Tails arising from the SOKE in figure 2 may be isolated by taking the same combination of signals as used to obtain trace (e) in figure 5. These traces are labelled as (b) and (d) in figure 6, and their shapes are indeed seen to be similar to those of traces (a) and (c) respectively. The difference of signals at $\phi= \pm 45^{\circ}$ should yield a tail that varies with pump polarization in the same manner as the SIFE, yet no reliable tail signal could be observed. Traces (a) and (c) in figure 6 have been fitted with equation (1). Trace (a) is well fitted by setting $C=0$. This yields a 


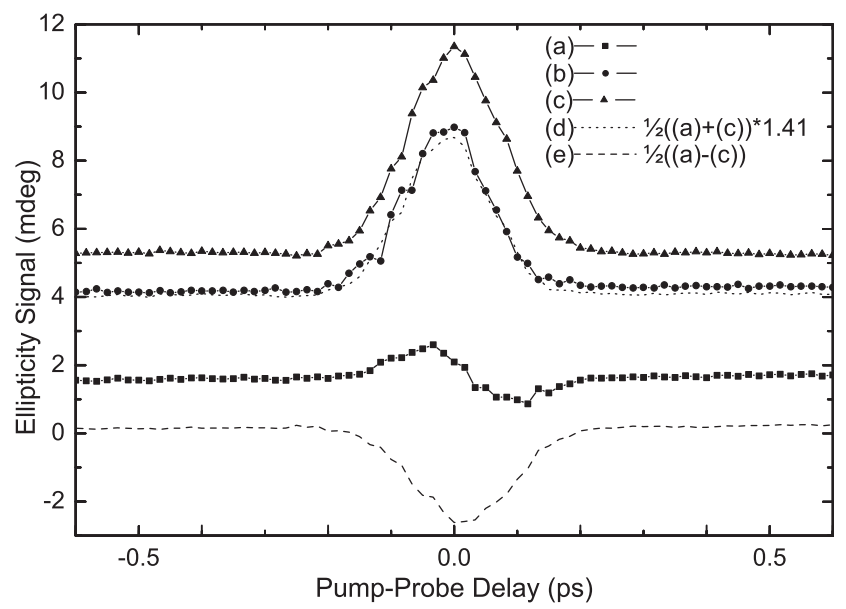

Figure 5. The shapes of the transient ellipticity signals near zero delay are shown for different pump polarizations in the second measurement configuration. The difference divided by two of the signals at (a) $\phi= \pm 22.5^{\circ}$, (b) $\phi= \pm 45^{\circ}$ and (c) $\phi= \pm 67.5^{\circ}$ are shown. For clarity the $45^{\circ}$ and the $67.5^{\circ}$ curves were shifted vertically upwards by 2 and 4 mdeg respectively. The SIFE (d), and SOKE (e) contributions extracted from (a) and (c) using equation (4) are also shown.

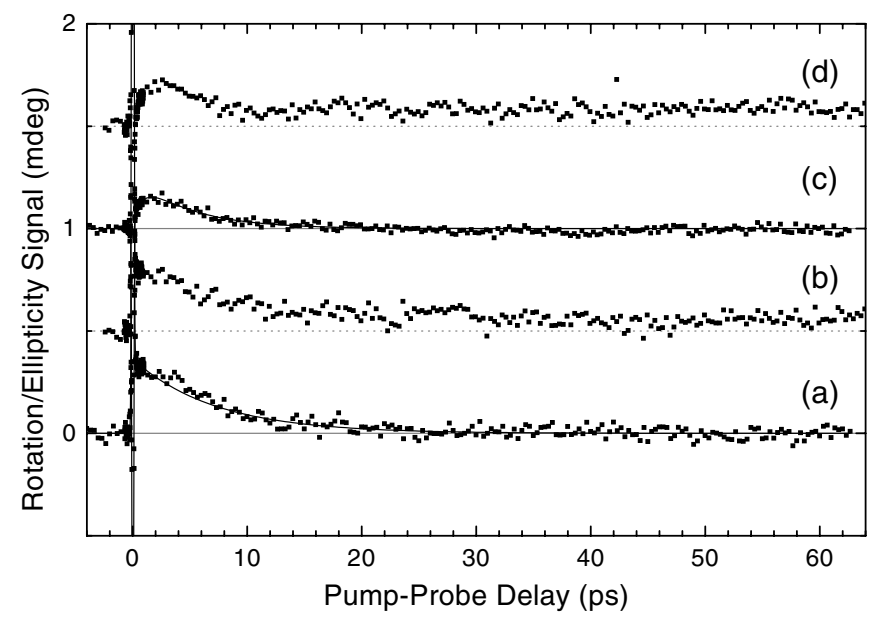

Figure 6. Combinations of traces from figures 1 and 2 are presented that isolate a tail signal with symmetry of the SOKE. Traces (a) and (c) show the rotation and ellipticity obtained from the difference divided by two of the traces at $\theta= \pm 45^{\circ}$ in figure 1 . Traces (b) and (d) show the rotation and ellipticity due to the SOKE obtained from figure 2 after performing the background subtraction described in the text. The shape of traces (b) and (d) is seen to be in good agreement with that of (a) and (c). The solid curves are fits to equation (1).

single relaxation time of $\tau_{1}=7 \mathrm{ps}$. For curve (c) it is necessary to retain both tail terms from equation (1). The fit yields relaxation times of $\tau_{1}=5 \mathrm{ps}$ and $\tau_{2}=0.8 \mathrm{ps}$, while the amplitudes of the two tail terms occur in the ratio $B / C=-1.2$.

\section{Discussion}

The peak at zero delay in the transient reflectivity signals presented in figures 1 and 2 has not been reported previously. The dependence of the peak height upon the pump polarization is 
similar to that of the SOKE peak and so the peak may be understood as a pump-induced linear dichroism in which the refractive index of the medium is modified in the direction parallel to the electric field of the pump. Our analysis has shown that the appearance of the peak in the rotation and ellipticity signals can be understood in terms of the coexistence of the SOKE and SIFE. Values have been deduced for the non-vanishing components of the cubic susceptibility tensor that describe the peak height. They have similar order of magnitude to those obtained previously for $\mathrm{Au}\left(\left|\chi_{x x y y}+\chi_{x y y x}\right| \approx 1.5 \times 10^{-8}\right.$ esu) by Zheludev and co-workers [15] from SIFE measurements. Since the scattering time for the electron momentum is very short, the peak shape is expected to correspond to the cross-correlation of the pump and probe pulse profiles, as assumed in equation (1). The slightly different pulse shapes deduced for the SIFE and SOKE in figure 5 are therefore unexpected. The angles of incidence used in the experiment imply that the time of arrival of the two edges of the focused laser beam are different by about 20 and 50 fs for the pump and probe respectively. This has the effect of slightly broadening the pulses. This geometrical effect is the same for the SIFE and SOKE and cannot explain the observed time shift in their peak values. In equation (1) we assumed that the first term, the Gaussian peak, was due to a process with a characteristic relaxation time that was negligible compared to the laser pulse width. However, as this characteristic relaxation time becomes significant, the peak must instead be described by a term of the same form as those used to describe the tails. For a relaxation time of a few tens of femtoseconds, that is still significantly smaller than the laser pulse width, the peak remains nearly symmetric with an FWHM similar to that of the Gaussian. However, the peak is delayed by a time that is approximately equal to the relaxation time. This suggests that the relaxation of the linear momentum in the SOKE is about 30 fs slower than that of the angular momentum in the SIFE. From a microscopic viewpoint the SIFE and SOKE peaks are associated with optically excited hot electrons and holes. Their initial energy distribution is determined by the band structure of the metal through the initial and final density of states and the selection rules for the pump transition. In the case of $\mathrm{Al}$ at $800 \mathrm{~nm}$, a continuous distribution of energies, extending to one photon energy from the Fermi level, is expected since excitation occurs between parallel bands [6]. The probe signal is determined by the same set of optical transitions and by the repopulation of states that results from energy and momentum relaxation of the hot electrons and holes. Therefore the relaxation times associated with the SIFE and SOKE peaks represent average values for the distribution of non-equilibrium carriers. They may be of direct relevance to the operation of hot electron devices where transport properties are determined both by momentum relaxation and the loss of carriers through inelastic scattering [16].

The rotation and ellipticity measurements made in the first experimental configuration, and the rotation measurements made in the second configuration, show clear tails. The dependence of their amplitude upon the pump polarization is similar to the SOKE and is characteristic of a pump-induced birefringence. There is no evidence for a SIFE-like tail that would indicate optical orientation of a long-lived spin population. In the first measurement configuration, the peak and tail have the same sign in the rotation measurements, but opposite sign in the ellipticity measurements. This suggests that the microscopic origin of the birefringence in the peak and tail signals is different, and supports our suggestion [12] that the tail is associated with an anisotropic deformation of the lattice while the peak is associated with the linear momentum of optically excited hot carriers. The relaxation of the tail occurs on timescales comparable to the time taken for the reflectivity signal to rise to its maximum value. It therefore seems likely that the decay of the tail is also associated with propagation of the acoustic transient into the interior of the sample. The lattice deformation provides a memory of the pump beam polarization which is sensed optically through the coupling of electrons to the lattice. This may explain why the relaxation time for the linear momentum is longer than that for the angular 
momentum in the case of the hot carriers. The second time constant of 0.8 ps observed in the ellipticity tail is comparable to the electron-phonon relaxation time of $0.6 \mathrm{ps}$, and may be associated with the changing population of states around the Fermi level as the electron system cools by transferring heat to the lattice.

In summary, the transient reflectivity, rotation and ellipticity of a thin film $\mathrm{Al}$ sample were studied as the polarization of the pump beam was systematically varied. The reflectivity signal was independent of the pump polarization apart from a peak at zero time delay due to a short-lived linear dichroism induced by the pump beam. The rotation and ellipticity signals generally contained a peak at zero time delay, with width comparable to that of the crosscorrelation of the laser pulse, and a longer-lived tail. The dependence of the peak amplitude upon the pump polarization can be understood in terms of a superposition of the SIFE and SOKE. This analysis has allowed us to determine the values of the relevant non-vanishing components of the cubic susceptibility tensor. By isolating the SIFE and SOKE contributions to the peaks, we deduced that the relaxation time for the SOKE may be somewhat longer than that for the SIFE in the ellipticity measurements. We interpret this in terms of a difference in the relaxation times for the linear and angular momentum of the excited carrier distribution. The amplitudes of the tails in the rotation and ellipticity signals both vary in a manner that is characteristic of the SOKE, supporting our view that a pump-induced linear birefringence results from an anisotropic lattice deformation. Two time constants were required to describe the ellipticity tail, suggesting that at least four time constants are required to fully describe the transient polarization response of aluminium.

\section{Acknowledgments}

The authors gratefully acknowledge discussions with V V Kruglyak, x-ray measurements performed by A T G Pym and Professor B K Tanner at the University of Durham, and the financial support of the Engineering and Physical Sciences Research Council (EPSRC).

\section{References}

[1] Brorson S D, Kazeroonian A, Moodera J S, Face D W, Cheng T K, Ippen E P, Dresselhaus M S and Dresselhaus G 1990 Phys. Rev. Lett. 642172

[2] Baumberg J J, Awschalom D D and Samarth N 1994 J. Appl. Phys. 756199

[3] Beaurepaire E, Merle J C, Daunois A and Bigot J Y 1996 Phys. Rev. Lett. 764250

[4] Saiki T, Takeuchi K, Kuwata-Gonokami M, Mitsuyu T and Ohkawa K 1992 Appl. Phys. Lett. 60192

[5] Svirko Y P and Zheludev N I 1998 Polarization of Light in Nonlinear Optics (Chichester: Wiley)

[6] Ashcroft N W and Sturm K 1971 Phys. Rev. B 31898

[7] Jiles D C 1984 Solid State Commun. 51327

[8] Richardson C J K 2000 Quantitative analysis of picosecond duration thermoelastic transients in polycrystalline metal thin films PhD Thesis Johns Hopkins University

[9] Tas G and Maris H J 1994 Phys. Rev. B 4915046

[10] Richardson C J K and Spicer J B 2002 Appl. Phys. Lett. 802895

[11] Guo C, Rodriguez G, Lobad A and Taylor A J 2000 Phys. Rev. Lett. 844493

[12] Wilks R, Hughes N D and Hicken R J 2003 J. Phys.: Condens. Matter 155129

[13] Meier F and Zakharchenya B P (ed) 1984 Optical Orientation (Amsterdam: North-Holland)

[14] Hohlfeld J, Wellershoff S S, Güdde J, Conrad U, Jähnke V and Matthias E 2000 Chem. Phys. 251237

[15] Zheludev N I, Bennett P J, Loh H, Popov S V, Shatwell I R, Svirko Y P, Gusev V E, Kamalov V F and Slobodchikov E V 1995 Opt. Lett. 201368

[16] Monsma D J, Lodder J C, Popma T J A and Dieny B 1995 Phys. Rev. Lett. 745260 